\title{
The Effects of the Short Foot Exercise on Navicular Drop: A Critically Appraised Topic
}

\author{
Cameron Haun, Cathleen N. Brown, Kimberly Hannigan, and Samuel T. Johnson
}

\begin{abstract}
Clinical Scenario: Deformation of the arch, as measured by navicular drop (ND), is linked to lower-extremity musculoskeletal injuries. The short foot exercise (SFE) has been used to strengthen the intrinsic foot muscles that support the arch. Clinical Question: Does the SFE decrease ND in healthy adults? Summary of Key Findings: Three studies that examined the use of the SFE on ND were included. A randomized control trial that compared the SFE to a towel-curl exercise and a control group found no significant differences between the 3 groups. A randomized control trial compared the SFE to the use of arch support insoles in individuals with a flexible flatfoot and found a significant improvement in the SFE group. A prospective cohort study, without a control group, reported a significant decrease in ND following a 4-week SFE intervention without a regression at an 8-week follow-up. Overall, two of the three studies reported a significant reduction in ND following an SFE. Clinical Bottom Line: There is preliminary data supporting the use of the SFE to decrease ND—particularly in individuals with a flexible flatfoot. However, issues with the study designs make it difficult to interpret the data. Strength of Recommendation: Due to limited evidence, there is grade B evidence to support the use of the SFE to decrease ND.
\end{abstract}

Keywords: clinical interventions, muscle activation, strength

\section{Clinical Scenario}

Excessive deformation of the arches of the foot is a concern for clinicians due to the resultant pathologic lower-extremity biomechanics. ${ }^{1}$ Multiple studies have found that excessive arch deformation measured by navicular drop (ND), a common clinical assessment, is linked to several lower-extremity overuse injuries, such as medial tibial stress syndrome and patellofemoral pain syndrome. ${ }^{2-6}$ The protracted recovery and high rates of recurrence of these injuries not only pose a burden for the patient, (eg, pain, decreased performance, lost time) but also require additional resources (eg, time, money) from the clinician. Therefore, clinicians need interventions that will not only treat the symptoms but also address the underlying causes of the condition in order to prevent recurrence.

Because the arches of the foot are supported by both static and dynamic structures (eg, bones, ligaments, aponeurosis, and muscles), rehabilitation for conditions associated with deformation of the arch have included static support, such as orthotics, and dynamic support through strengthening of the muscles. One muscle group of particular interest is the intrinsic foot muscles, due to their role as an active stabilizer to the arches of the foot. ${ }^{7}$ An approach we have observed being utilized for overuse injuries associated with deformation of the arch is the short foot exercise (SFE). The goal of the SFE is to support the arches of the foot, specifically the medial longitudinal arch, by isometrically activating the intrinsic foot muscles. ${ }^{7,8}$ To perform the SFE, an individual is instructed to raise the medial longitudinal arch by bringing the metatarsal heads toward the heel without flexing the toes. ${ }^{9-11}$ There is evidence that

Haun is with Providence Sports Medicine, Portland, OR, USA. Brown, Hannigan, and Johnson are with the College of Public Health and Human Sciences, Oregon State University, Corvallis, OR, USA. Johnson (Sam.johnson@oregonstate.edu) is corresponding author. the SFE results in increased dynamic balance control ${ }^{12}$ and somatosensory function ${ }^{13}$ and greater activation of the intrinsic foot muscles compared with other common exercises. ${ }^{8}$ Therefore, a rehabilitation plan that incorporates the SFE may be effective in supporting the arch and resolving the condition. Unfortunately, to our knowledge, there has not been any research examining the use of the SFE in the treatment of specific conditions related to excessive arch deformation, such as medial tibial stress syndrome and patellofemoral pain syndrome. This makes it challenging for clinicians to judge the effectiveness of SFE in treating these conditions. However, because ND is a risk factor for several overuse injuries associated with arch deformation, a finding that the SFE is able to improve ND may provide initial evidence for the utility of the SFE in the prevention and treatment of these conditions.

\section{Clinical Question}

Does the SFE decrease ND in healthy adults?

\section{Summary of Search, Best Evidence Appraised, and Key Findings}

- The literature was searched for studies of level 2 evidence or higher ${ }^{14}$ that investigated the effects of the SFE on ND.

- The literature search returned 8 possible studies related to the clinical question. Three studies met the inclusion criteria: 2 randomized control trials ${ }^{9,10}$ and 1 prospective cohort. ${ }^{11}$

- Lynn et $\mathrm{al}^{9}$ performed a randomized control trial comparing ND in healthy young adults after a 4-week intervention of SFE, towel-curl exercises, or a control group. They reported no significant differences between groups for either the dominant or nondominant leg. 
- Kim and $\mathrm{Kim}^{10}$ compared the ND of individuals with a flexible flatfoot following either a 5-week SFE intervention or an intervention consisting of wearing custom insoles 3 times a week for 30-minute intervals for the same 5 weeks. They reported a decrease in ND in the SFE group after the intervention but not in the insole group. In addition, the comparison of change scores between the groups indicated a significant difference between groups, with the SFE having a greater reduction in ND.

- Mulligan and Cook $^{11}$ measured ND in a cohort of healthy young adults following a 4-week SFE intervention. There was a significant decrease at the end of the intervention that was retained during a follow-up at 8 weeks. A post hoc analysis indicated that participants with an ND drop greater than $15 \mathrm{~mm}$ (ie, flexible flatfoot) demonstrated a greater improvement. However, there was no control or comparison group included in the study.

- Two of the 3 studies reported a decrease in ND following an SFE intervention. While the studies used different intervention volumes and progressions, the SFE instructions were consistent across the publications. This suggests that SFE may improve ND. However, the 2 studies with positive results either did not include or had an inadequate control group and also used statistical analyses that may have been inappropriate. Furthermore, no studies have directly assessed SFE in individuals with conditions associated with arch deformation. Therefore, the evidence should be considered preliminary.

\section{Clinical Bottom Line}

Preliminary evidence suggests that SFE improves ND in healthy adults, particularly in those with a flexible flatfoot. The evidence should be considered preliminary due to concerns related to the methods used in the studies. Specifically, the 2 studies with a positive outcome either had no control group, or the comparison group was inadequate to determine if the results were due specifically to the SFE intervention. In addition, the 3 studies included in the critically appraised topic (CAT) studied participants that were asymptomatic. However, available evidence suggests there are other positive benefits of the SFE, including increased dynamic balance control, ${ }^{12}$ improved somatosensory function in ankle instability, ${ }^{13}$ and greater activation of the intrinsic foot muscles. ${ }^{8}$ Clinicians should use their best clinical judgment when deciding whether to utilize the SFE as an intervention for decreasing ND.

\section{Strength of Recommendation}

Due to inconsistent results in the studies used, there is grade B evidence to support the use of SFE to decrease ND.

\section{Search Strategy}

\section{Terms Used to Guide Search Strategy}

- Patient group: Healthy adults

- Intervention: SFE

- Comparison: Control group or pretest/posttest

- Outcome: ND

\section{Sources of Evidence Searched}

- Medline

- EBSCOhost

- CINAHL

- Cochrane Database

- SPORTDiscus

- Additional sources obtained via review of reference lists and hand search

\section{Inclusion and Exclusion Criteria}

\section{Inclusion Criteria}

- Studies that investigated the use of the SFE

- Studies that used ND

- Level 2 evidence or higher ${ }^{14}$

- Limited to English language

- Limited to the past 10 years (2008-2018)

- Indexed in one of the above databases

\section{Exclusion Criteria}

- Studies that did not use the SFE as an intervention

- Studies that did not use ND

\section{Results of Search}

Three relevant studies $^{9-11}$ were located and categorized as described in Figure 1 and Tables 1 and 2 (based on Levels of Evidence, Oxford Centre for Evidence Based Medicine, $2009^{14}$ ).

\section{Best Evidence}

The studies in Tables 1 and 2 were identified as the best evidence and selected for inclusion in this CAT.

Initial database search: $\mathrm{N}=41$

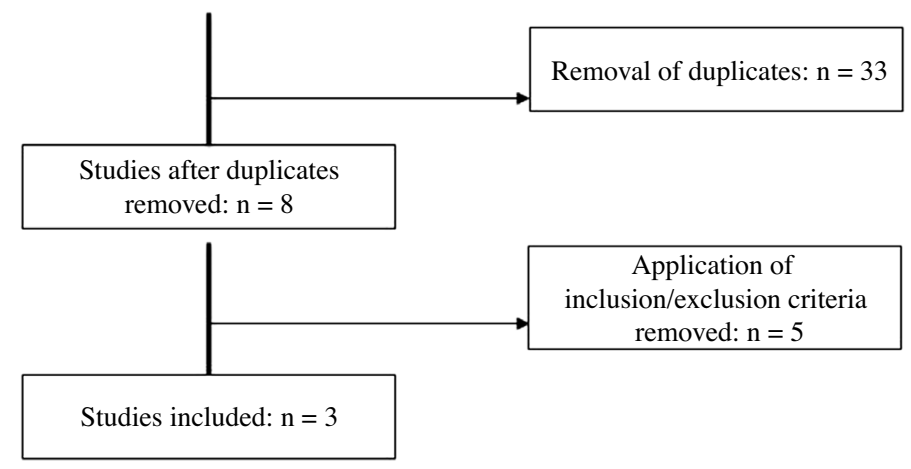

Figure 1 - Summary of the article screening process. 
Haun et al

Table 1 Summary of Study Designs

\begin{tabular}{llcl}
\hline Level of evidence & Study design/methodology of articles retrieved & Number located & Reference \\
\hline 2 & Randomized control trial & 2 & ${\text { Lynn et } \mathrm{al}^{9}}^{10}$ \\
2 & Prospective cohort & 1 & Mim Kim Kim $^{10}$ \\
& & & Mulligan and Cook \\
\hline
\end{tabular}

\section{Implications for Practice, Education, and Future Research}

The results of this CAT indicate preliminary evidence that the SFE intervention has a positive effect on ND. However, this result should be considered with caution due to important methodological differences in the studies and also due to the fact the clinically meaningful difference in ND is currently unknown (although an ND greater than 10-15 $\mathrm{mm}$ has been considered excessive. ${ }^{15,16}$ )

The Mulligan and Cook $^{11}$ study found a significant difference in ND following a 4-week intervention that was retained after a 4-week retention period. Unfortunately, effect sizes were not reported, so it is unknown if the $1.8 \mathrm{~mm}$ (4-wk ND minus preND) and $2.2 \mathrm{~mm}$ (8-wk ND minus pre-ND) decreases were clinically meaningful. However, in the discussion of the study, they reported that a post hoc analysis indicated the intervention had a greater impact on participants with a ND greater than $15 \mathrm{~mm}$. It is important to note that the study did not include a control group, so it is unknown if the change in ND was due to the SFE, to the activation of the intrinsic foot muscles regardless of the exercise, or to a learning effect. In addition, the researchers included both limbs in the one-way repeated-measures analysis of variance without accounting for any issues of independence (an assumption of an analysis of variance).

The other study that reported a statistically significant difference in ND following an SFE intervention was the Kim and $\mathrm{Kim}^{10}$ study. This study was unique among the 3 studies included in the CAT, in that the participants all had an asymptomatic flexible flatfoot (ND > $10 \mathrm{~mm}$ ). The researchers did include a comparison group. Specifically, participants in the comparison group wore arch support insoles for the same volume of time that the SFE participants performed the exercise (ie, 30-min sessions, 3 times per wk, for $5 \mathrm{wk}$ ). The SFE group had a 3.7-mm decrease in ND, and the insole group had a 1.7-mm decrease. Like the Mulligan and Cook ${ }^{11}$ study, the effect sizes were not reported. In addition, while there was a comparison group included in the study, the focus of the 2 interventions was different-one focused on activation of the intrinsic foot muscles (SFE group), and the other focused on providing structural support through insoles. Therefore, it is difficult to know whether the improved ND in the SFE group was due specifically to the SFE or to the fact that one intervention targeted activation of a specific muscle group whereas the other did not. In other words, other exercises that target the intrinsic foot muscles could be just as effective. In addition, the statistical design (ie, multiple $t$ tests instead of a repeated-measures analysis of variance) make interpretation of the study results challenging for the clinician.

The Lynn et al $^{9}$ study did account for the fact there are multiple exercises with the potential to activate the foot intrinsic muscles. In addition to the SFE group, they included a towel-curl exercise group and a control group. There were no significant interactions or main effects for any intervention on either the dominant or the nondominant limb. They reported a $1.8-\mathrm{mm}$ decrease in navicular height on the dominant limb and a $1.3-\mathrm{mm}$ decrease on the nondominant limb. While the differences were not statistically significant, the effect sizes for the dominant and nondominant limbs were -0.56 and -0.38 , respectively $\left(\right.$ effect size $\left.=\frac{\text { post mean-pre mean }}{\text { pre standard deviation }}\right)$. The towel-curl group had no change (effect size $=0.00$ ) in the dominant limb and a $2.1-\mathrm{mm}$ increase $($ effect size $=0.26$ ) in the nondominant limb. The control group had a $0.4-\mathrm{mm}$ decrease (effect size $=-0.06$ ) and a $0.3-\mathrm{mm}$ decrease (effect size $=-0.05$ ) for the dominant and nondominant limbs, respectively. Taken together, although there was not a statistically significant difference between groups, the reported effect size for the SFE group could be considered moderate.

If clinicians decide to prescribe SFE, they may be interested in the SFE prescription in the studies. All 3 studies taught the SFE with the same instructions. In addition, the intervention length was relatively consistent across studies (ie, a 4- or 5-wk program). However, other elements of the exercise prescription varied between studies. Two studies had the participants perform the exercise daily, ${ }^{9,11}$ and the other study asked participants to complete the exercise 3 days per week. ${ }^{10}$ The duration of the contractions were 5 to 10 seconds in all 3 studies, but the repetitions per session varied between 30 and 120. Taken together, this resulted in exercise volumes that ranged from 4200 to 18,000 seconds of contraction across the interventions. Interestingly, the 2 studies that reported a significant reduction in ND utilized the lowest and highest exercise volumes of the 3 studies. This indicates that the specific exercise volume is perhaps not as important as simply prescribing SFE in order to activate the intrinsic foot muscles.

Clinicians may also be interested in exercise progression. While all 3 of the studies began the SFE program with the participant in the seated position, the progression was different in each. The Kim and $\mathrm{Kim}^{10}$ study utilized the seated position throughout the 5-week intervention and reported significant results. The Lynn et $\mathrm{al}^{9}$ study instructed participants to progress to a standing position for the last 2 weeks of the intervention but did not find a significant change in navicular height. The Mulligan and Cook $^{11}$ study, which also reported a significant decrease in ND, instructed participants to self-advance from a seated position to a double-leg stance and eventually a single-leg stance when they were able to complete the full 3-minute session without increased soreness the following day. Participants in that study were also instructed to alter their visual status (open to closed) and change the stability of the surface (stable to unstable) that the SFE was performed on; however, it was not clearly indicated how these progressions were implemented. ${ }^{11}$ Again, as with exercise volume, statistical significance did not seem to correspond to the demands of the progression. In other words, the study that did not include any exercise progression and the one with the most demanding progression both reported a significant reduction in ND. This suggests that exercise progression to more demanding positions may be unnecessary.

In conclusion, preliminary evidence suggests that SFE interventions may improve ND, with the greatest potential benefit to individuals with a flexible flatfoot. However, this should be 


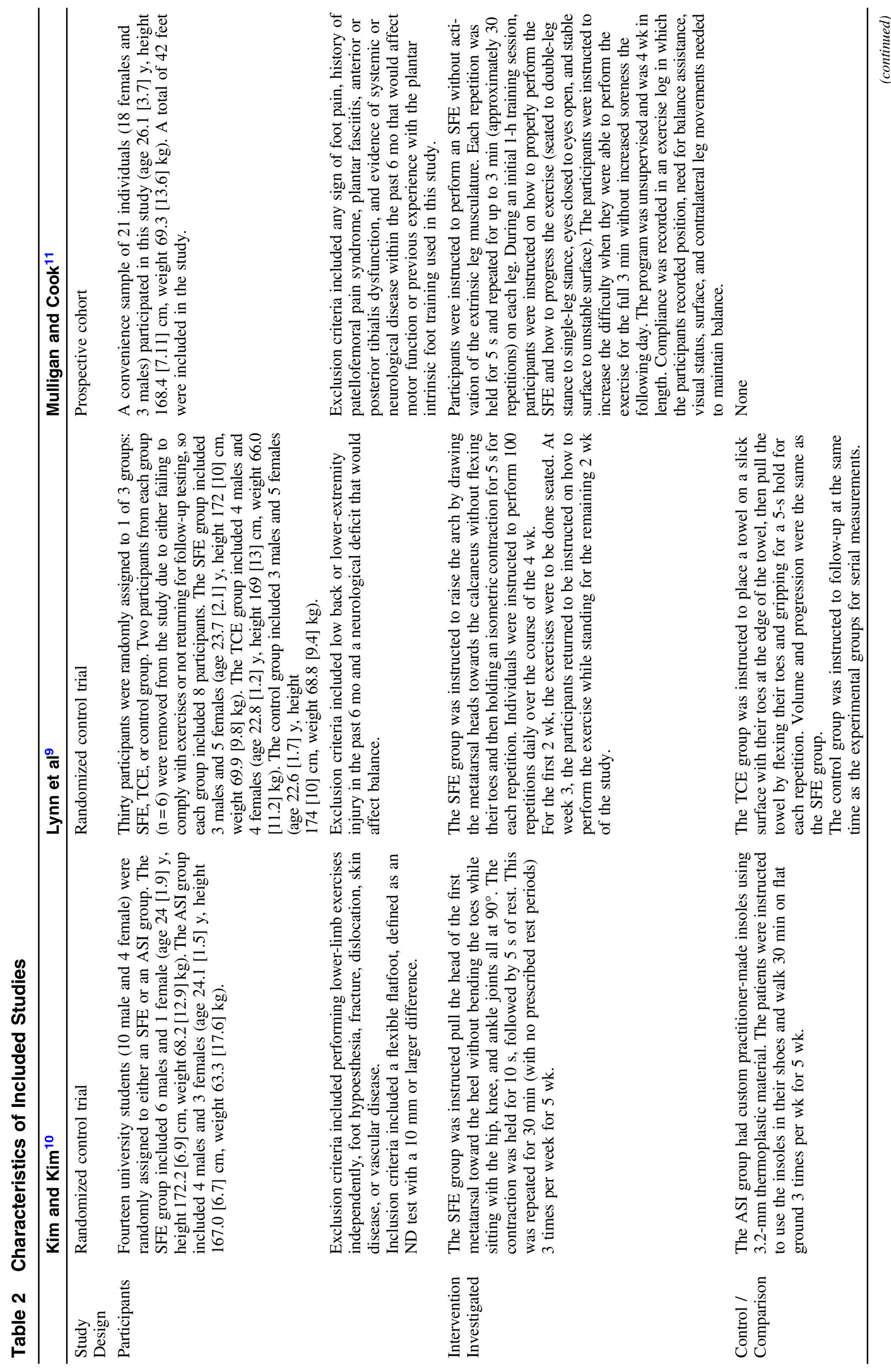




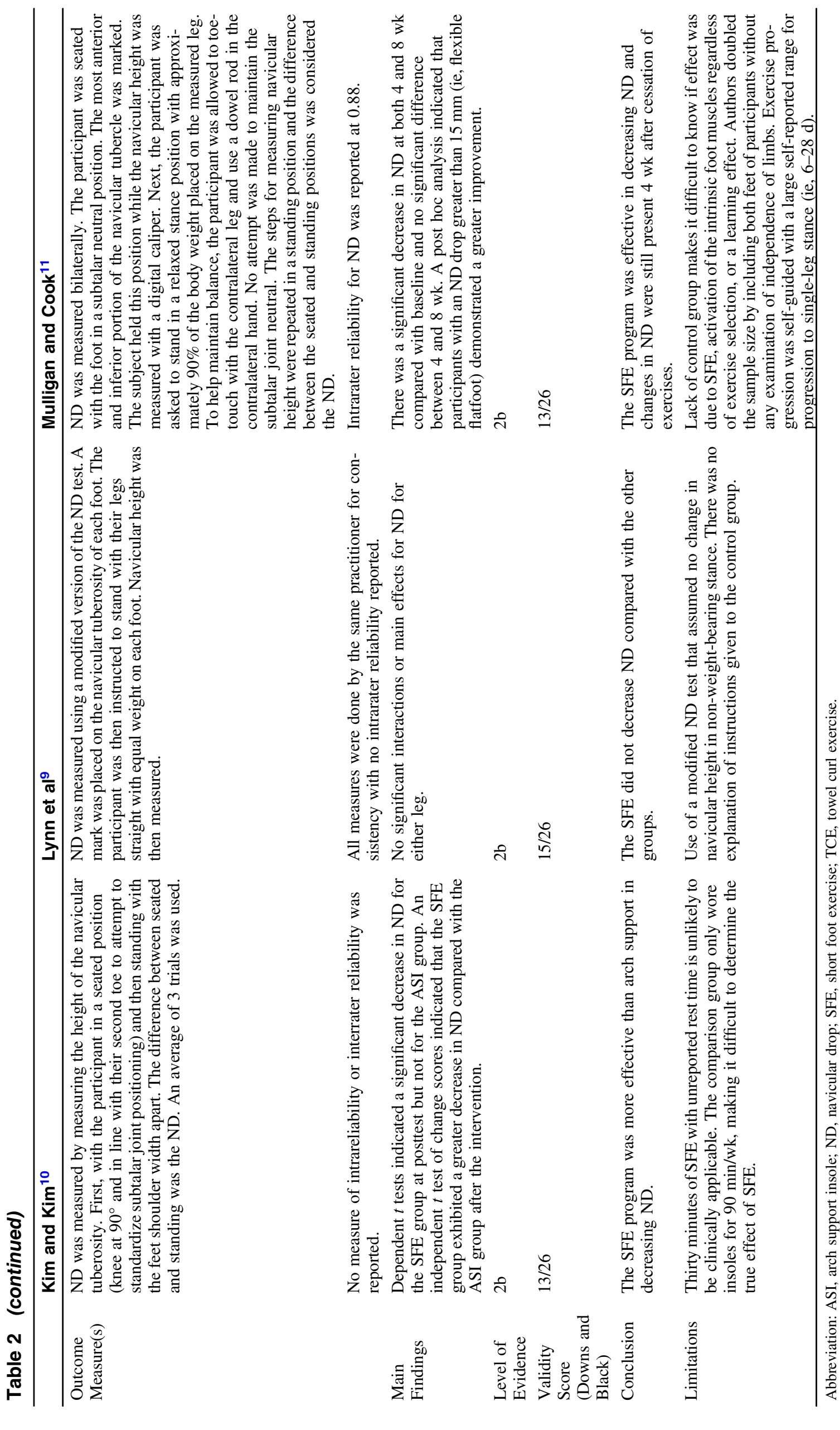

JSR Vol. 30, No. 1, 2021 
interpreted with caution due to the aforementioned concerns. Specifically, the design of the 2 studies that showed a reduction in ND make it difficult to determine if the effects were specifically due to the SFE intervention. Future studies should utilize comparison and control groups in order to help answer this question. In addition, studying symptomatic individuals would also assist the clinician. Finally, future research could be performed to determine if a specific progression and/or threshold of exercise volume is needed. This CAT should be reviewed in 2 years to determine whether there is additional evidence that could change the clinical bottom line.

\section{References}

1. Van Boerum DH, Sangeorzan BJ. Biomechanics and pathophysiology of flat foot. Foot Ankle Clin. 2003;8(3):419-430.

2. Newman P, Witchalls J, Waddington G, Adams R. Risk factors associated with medial tibial stress syndrome in runners: a systematic review and meta-analysis. Open Access J Sports Med. 2013;4:229241. PubMed ID: 24379729 doi:10.2147/OAJSM.S39331

3. Reinking MF, Austin TM, Richter RR, Krieger MM. Medial tibial stress syndrome in active individuals: a systematic review and metaanalysis of risk factors. Sports Health. 2017;9(3):252-261. doi:10. $1177 / 1941738116673299$

4. Hamstra-Wright KL, Bliven KCH, Bay C. Risk factors for medial tibial stress syndrome in physically active individuals such as runners and military personnel: a systematic review and meta-analysis. $\mathrm{Br} J$ Sports Med. 2015;49(6):362-369. PubMed ID: 25185588 doi:10. 1136/bjsports-2014-093462

5. Winkelmann ZK, Anderson D, Games KE, Eberman LE. Risk factors for medial tibial stress syndrome in active individuals: an evidencebased review. J Athl Train. 2016;51(12):1049-1052. PubMed ID: 27835043 doi:10.4085/1062-6050-51.12.13

6. Boling MC, Padua DA, Marshall SW, Guskiewicz K, Pyne S, Beutler A. A prospective investigation of biomechanical risk factors for patellofemoral pain syndrome: the Joint Undertaking to Monitor and Prevent ACL Injury (JUMP-ACL) cohort. Am J Sports Med.
2009;37(11):2108-2116. PubMed ID: 19797162 doi:10.1177/ 0363546509337934

7. McKeon PO, Fourchet F. Freeing the foot: integrating the foot core system into rehabilitation for lower extremity injuries. Clin Sports Med. 2015;34(2):347-361. PubMed ID: 25818718 doi:10.1016/j. csm.2014.12.002

8. Gooding TM, Feger MA, Hart JM, Hertel J. Intrinsic foot muscle activation during specific exercises: a T2 time magnetic resonance imaging study. J Athl Train. 2016;51(8):644-650. PubMed ID: 27690528 doi:10.4085/1062-6050-51.10.07

9. Lynn SK, Padilla RA, Tsang KKW. Differences in static- and dynamic-balance task performance after 4 weeks of intrinsic-footmuscle training: the short-foot exercise versus the towel-curl exercise. J Sport Rehabil. 2012;21(4):327-333. PubMed ID: 22715143

10. Kim E-K, Kim JS. The effects of short foot exercises and arch support insoles on improvement in the medial longitudinal arch and dynamic balance of flexible flatfoot patients. J Phys Ther Sci. 2016;28(11): 3136-3139. PubMed ID: 27942135 doi:10.1589/jpts.28.3136

11. Mulligan EP, Cook PG. Effect of plantar intrinsic muscle training on medial longitudinal arch morphology and dynamic function. Man Ther. 2013;18(5):425-430. PubMed ID: 23632367 doi:10.1016/j. math.2013.02.007

12. Mignogna CA, Welsch LA, Hoch MC. The effects of short-foot exercises on postural control: a critically appraised topic. Int J Athl Ther Train. 2016;21(6):8-12. doi:10.1123/ijatt.2016-0049

13. Lee E, Cho J, Lee S. Short-foot exercise promotes quantitative somatosensory function in ankle instability: a randomized controlled trial. Med Sci Monit. 2019;25:618-626. doi:10.12659/MSM.912785

14. CEBM. Oxford Centre for Evidence-based Medicine-Levels of Evidence (March 2009). June 11, 2009. https://www.cebm.net/ 2009/06/oxford-centre-evidence-based-medicine-levels-evidencemarch-2009/. Accessed June 16, 2019.

15. Mueller MJ, Host JV, Norton BJ. Navicular drop as a composite measure of excessive pronation. J Am Podiatr Med Assoc. 1993; 83(4):198-202. doi:10.7547/87507315-83-4-198

16. Brody DM. Techniques in the evaluation and treatment of the injured runner. Orthop Clin North Am. 1982;13(3):541-558. PubMed ID: 6124922 